\title{
6p22.3 amplification as a biomarker and potential therapeutic target of advanced stage bladder cancer
}

\author{
He Shen ${ }^{1, *}$, Carl D. Morrison ${ }^{2, *}$, Jianmin Zhang ${ }^{1}$, Willie Underwood III $^{3}$, Nuo Yang \\ 1, Costa Frangou ${ }^{1}$, Kevin Eng ${ }^{4}$, Karen Head ${ }^{2}$, Roni J. Bollag7, Sravan K. Kavuri', \\ Amyn M. Rojiani7, Yingwei Li ${ }^{1}$, Li Yan ${ }^{4}$, Annette Hill ${ }^{2}$, Anna Woloszynska-Read ${ }^{5}$, \\ Jianmin Wang ${ }^{4}$, Song Liu ${ }^{4}$, Donald L. Trump ${ }^{6}$ \& Candace S. Johnson ${ }^{5}$ \\ ${ }^{1}$ Department of Cancer Genetics, Roswell Park Cancer Institute, Buffalo, NY 14263 \\ 2 Department of Pathology, Roswell Park Cancer Institute, Buffalo, NY 14263 \\ ${ }^{3}$ Department of Urology, Roswell Park Cancer Institute, Buffalo, NY 14263 \\ ${ }^{4}$ Center for Biostatistics, Roswell Park Cancer Institute, Buffalo, NY 14263 \\ ${ }^{5}$ Department of Pharmacology \& Therapeutics, Roswell Park Cancer Institute, Buffalo, NY 14263 \\ ${ }^{6}$ Department of Medicine, Roswell Park Cancer Institute, Buffalo, NY 14263 \\ 7 Department of Pathology, Georgia Regents University, Augusta, GA \\ *Equal contribution \\ Correspondence to: Jianmin Zhang, email: jianmin.zhang@roswellpark.org \\ Keywords: bladder cancer, chromosome 6p22, FISH, outcome, survival \\ Received: September 9, $2013 \quad$ Accepted: October 29, $2013 \quad$ Published: October 29, 2013
}

This is an open-access article distributed under the terms of the Creative Commons Attribution License, which permits unrestricted use, distribution, and reproduction in any medium, provided the original author and source are credited.

\section{ABSTRACT:}

Genetic and epigenetic alterations have been identified as to contribute directly or indirectly to the generation of transitional cell carcinoma of the urinary bladder (TCC-UB). In a comparative fashion much less is known about copy number alterations in TCC-UB, but it appears that amplification of chromosome $6 \mathrm{p2} 2$ is one of the most frequent changes. Using fluorescence in situ hybridization (FISH) analyses, we evaluated chromosomal 6p22 amplification in a large cohort of bladder cancer patients with complete surgical staging and outcome data. We have also used shRNA knockdown candidate oncogenes in the cell based study. We found that amplification of chromosome 6 p22.3 is significantly associated with the muscle-invasive transitional cell carcinoma of the urinary bladder (TCC-UB) $(22 \%)$ in contrast to superficial TCCUB $(9 \%) \quad(p=7.2-04)$. The rate of $6 p 22.3$ amplification in $p N>1$ patients $(32 \%)$ is more than twice that in pNO $(16 \%)$ patients $(p=0.05)$. Interestingly, we found that 6p22.3 amplification is as twice as high $(p=0.0201)$ in African American (AA) than European American (EA) TCC-UB patients. Moreover, we showed that the expression of some candidate genes (E2F3, CDKAL1 and Sox4) in the 6p22.3 region is highly correlated with the chromosomal amplification. In particular, knockdown of E2F3 inhibits cell proliferation in a 6p22.3-dependent manner, whereas knockdown of CDKAL1 and Sox 4 has no effect on cell proliferation. Using gene expression profiling, we further identified some common as well as distinctive subset targets of the E2F3 family members. In summary, our data indicate that E2F3 is a key regulator of cell proliferation in a subset of bladder cancer and the 6p22.3 amplicon is a biomarker of aggressive phenotype in this tumor type.

\section{INTRODUCTION}

Many genetic and epigenetic alterations have been identified as to contribute directly or indirectly to the generation of transitional cell carcinoma of the urinary bladder (TCC-UB). Genetic alterations occurring in low- 
grade superficial TCC-UB are most frequently caused by activating mutations of proto-oncogenes, of which fibroblast growth factor receptor 3 (FGFR3) and HRAS are most prevalent, with mutations in up to $75 \%$ and $30 \%$ of the papillary tumors, respectively $[1,2]$. Since both these oncogenes activate the RAS/MEK/ERK signaling pathway, they appear to be mutually exclusive [3]. In contrast, the majority of muscle-invasive TCC-UB arises through inactivation of the tumor suppressor pathways of TP53, RB1 or PTEN [1, 4]. These mutations result in genomic instability and an anti-apoptotic phenotype, which enables tumor progression through accumulation of mutations. Other mutations that are observed in both subsets of TCC-UB include mutations of phosphoinositide 3-kinase (PI3K, $>10 \%$ ) and deletion of the tumor suppressor genes Tuberous Sclerosis 1 (TSC1, 10\%), Patched (PTCH, 40\% LOH), CDKN2A, and Deleted in Bladder Cancer 1 (DBC1, 50\%) $[5,6]$.

In a comparative fashion much less is known about copy number alterations in TCC-UB, but it appears that amplification of chromosome $6 \mathrm{p} 22$ is one of the most frequent changes $[7,8]$. In the present study, using The Cancer Genome Atlas (TCGA) dataset and cBio Cancer Genomics Portal analysis, we found that chromosome

A.

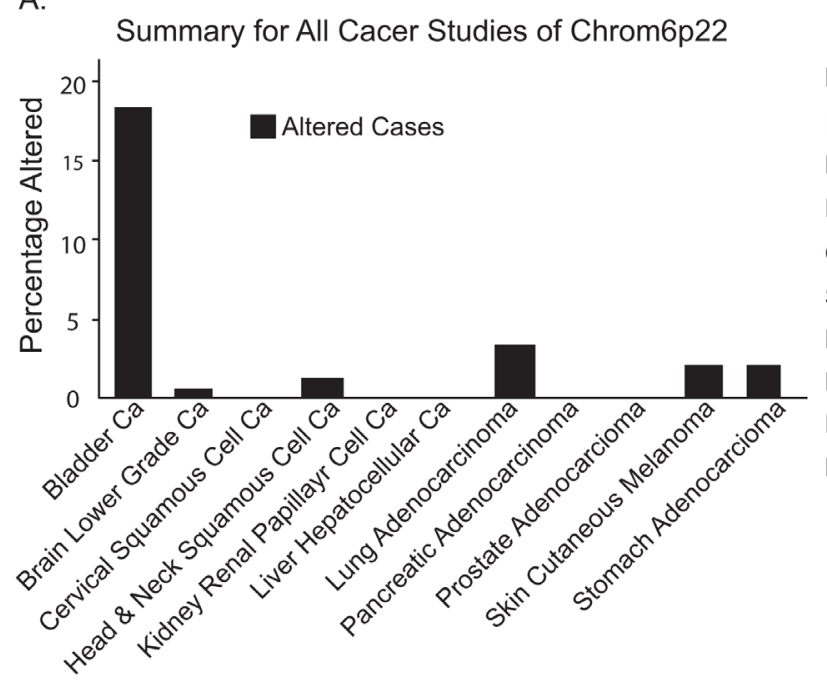

C.
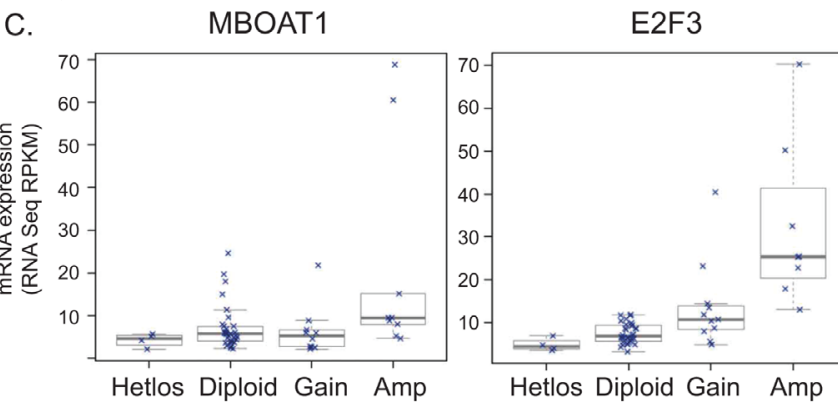

Putative Copy-number alterations from GISTIC
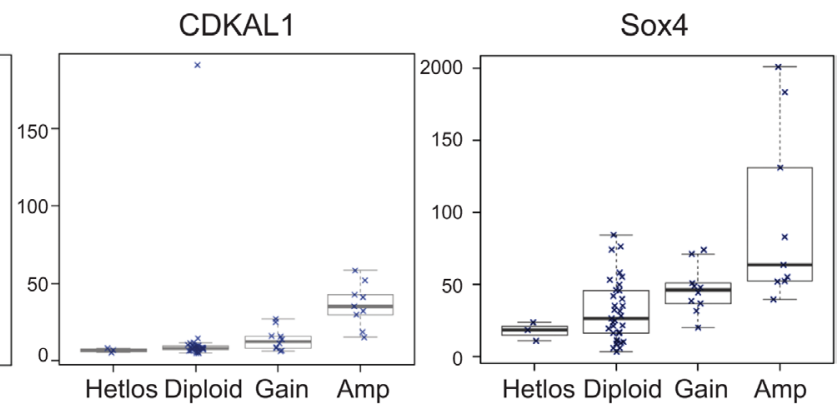

6p22 was highly amplified in bladder cancer patients $(18 \%)$ in comparison to other carcinomas. In our cohort of 365 TCC-UB, we found that amplification of chromosome 6 p22 was significantly associated with muscle-invasive TCC-UB (22\%) in contrast to superficial TCC-UB (9\%) $(\mathrm{p}=7.2-04)$. The rate of $6 \mathrm{p} 22.3$ amplification in $\mathrm{pN}>1$ patients $(32 \%)$ is more than twice that in pN0 $(16 \%)$ patients $(\mathrm{p}=0.05)$. Interestingly, we found that $6 \mathrm{p} 22$ amplification is as twice as high $(\mathrm{p}=0.0201)$ in African American (AA) than European American (EA) TCC-UB patients. We further characterized E2F3 as a major cell proliferation effector of $6 \mathrm{p} 22$ amplification and identified distinct target genes regulated by the E2F3 family members.

\section{RESULTS}

\section{Chromosomal 6p22 amplification in TCGA and RPCI bladder cancer patient.}

With the rapidly declining cost of next-generation sequencing and major national and international efforts

B.

ID4

MBOAT1

E2F3

CDKAL1

Sox4

LINC00340

PRL

HDGFL1

LOC100996507 0\%

I Amplification

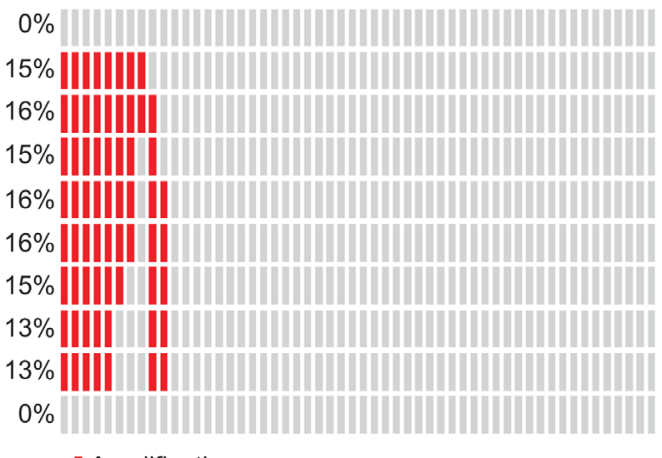

Diploid Gain Amp

Figure 1: Chromosomal 6p22 amplification in bladder cancer patients. A) Data analysis of The Cancer Genome Atlas (TCGA), indicating high prevalence of chromosomal 6p22 amplification in bladder cancer patients. B) High resolution analysis of DNA copy number gains of chromosome 6p22 in bladder cancer in TCGA data. C) Correlation between chromosomal 6p22 amplification and MBOAT1, E2F3, CDKAL1 and Sox4 expression by RNAseq. 


\begin{tabular}{|c|c|c|c|c|c|c|c|c|c|}
\hline \multicolumn{10}{|c|}{ Table 1: Clinicopathological Features of Patients with 6p22.3 Amplification. } \\
\hline & & \multicolumn{4}{|c|}{$\begin{array}{l}325 \text { White } \\
\text { and 1 Asian }\end{array}$} & \multicolumn{4}{|c|}{$\begin{array}{l}38 \text { African } \\
\text { American }\end{array}$} \\
\hline & & \multicolumn{2}{|c|}{ Total } & \multicolumn{2}{|c|}{$\begin{array}{c}6 \mathrm{p} \\
\text { Amplified }\end{array}$} & \multicolumn{2}{|c|}{ Total } & \multicolumn{2}{|c|}{$\begin{array}{c}6 \mathrm{p} \\
\text { Amplified }\end{array}$} \\
\hline & & \# & $\%$ & \# & $\%$ & \# & $\%$ & \# & $\%$ \\
\hline Total Patients & & 326 & $100 \%$ & 50 & $15 \%$ & 38 & $100 \%$ & 12 & $32 \%$ \\
\hline \multirow[t]{2}{*}{ Sex } & Female & 70 & $21 \%$ & 8 & $11 \%$ & 17 & $45 \%$ & 5 & $29 \%$ \\
\hline & Male & 256 & $79 \%$ & 42 & $16 \%$ & 21 & $55 \%$ & 7 & $33 \%$ \\
\hline \multirow[t]{2}{*}{ Age } & $<60$ & 66 & $20 \%$ & 9 & $14 \%$ & 15 & $39 \%$ & 6 & $40 \%$ \\
\hline & $>60$ & 260 & $80 \%$ & 41 & $16 \%$ & 23 & $61 \%$ & 6 & $26 \%$ \\
\hline \multirow[t]{3}{*}{ Smoking History } & $\begin{array}{l}\text { Current or previous } \\
\text { smoker }\end{array}$ & 268 & $82 \%$ & 42 & $16 \%$ & 7 & $18 \%$ & 4 & $57 \%$ \\
\hline & Never smoked & 58 & $18 \%$ & 8 & $14 \%$ & 2 & $5 \%$ & 0 & $0 \%$ \\
\hline & Unknown & 2 & $1 \%$ & 0 & $0 \%$ & 30 & $79 \%$ & 8 & $27 \%$ \\
\hline \multirow[t]{2}{*}{ Bladder Cancer } & Non-muscle invasive & 118 & $36 \%$ & 10 & $8 \%$ & 19 & $50 \%$ & 3 & $16 \%$ \\
\hline & Muscle invasive & 208 & $64 \%$ & 40 & $19 \%$ & 19 & $50 \%$ & 9 & $47 \%$ \\
\hline \multirow[t]{2}{*}{$\begin{array}{l}\text { Superficial Bladder } \\
\text { Cancer Grade }\end{array}$} & Low grade & 40 & $12 \%$ & 1 & $3 \%$ & 7 & $18 \%$ & 0 & $0 \%$ \\
\hline & High grade & 76 & $23 \%$ & 9 & $12 \%$ & 17 & $45 \%$ & 3 & $18 \%$ \\
\hline \multirow[t]{4}{*}{$\begin{array}{l}\text { Superficial Bladder } \\
\text { Cancer Stage } \\
\end{array}$} & $0 \mathrm{a}$ & 62 & $19 \%$ & 3 & $5 \%$ & 11 & $29 \%$ & 0 & $0 \%$ \\
\hline & 0is & 2 & $1 \%$ & 0 & $0 \%$ & 1 & $3 \%$ & 1 & $100 \%$ \\
\hline & $\mathrm{I}$ & 42 & $13 \%$ & 5 & $12 \%$ & 7 & $18 \%$ & 2 & $29 \%$ \\
\hline & IV & 12 & $4 \%$ & 2 & $17 \%$ & 0 & & & \\
\hline \multirow[t]{4}{*}{ Nodal Status MIBC } & $\mathrm{pN} 0$ & 107 & $33 \%$ & 16 & $15 \%$ & 5 & $13 \%$ & 2 & $40 \%$ \\
\hline & $\mathrm{pN} 1$ & 35 & $11 \%$ & 6 & $17 \%$ & 3 & $8 \%$ & 2 & $67 \%$ \\
\hline & $\mathrm{pN}>1$ & 41 & $13 \%$ & 15 & $37 \%$ & 6 & $16 \%$ & 4 & $67 \%$ \\
\hline & Unknown & 0 & & & & 5 & $13 \%$ & 1 & $20 \%$ \\
\hline \multirow[t]{3}{*}{$\begin{array}{c}\text { Muscle Invasive } \\
\text { Bladder Cancer Stage }\end{array}$} & II & 45 & $14 \%$ & 6 & $13 \%$ & 6 & $16 \%$ & 1 & $17 \%$ \\
\hline & III & 64 & $20 \%$ & 13 & $20 \%$ & 6 & $16 \%$ & 2 & $33 \%$ \\
\hline & IV & 99 & $30 \%$ & 21 & $21 \%$ & 7 & $18 \%$ & 6 & $86 \%$ \\
\hline \multirow[t]{3}{*}{ Outcome MIBC } & $\begin{array}{l}\text { Evidence Of This } \\
\text { Cancer }\end{array}$ & 98 & $30 \%$ & 18 & $18 \%$ & 4 & $11 \%$ & 2 & $50 \%$ \\
\hline & \begin{tabular}{|c|}
$\begin{array}{c}\text { No Evidence Of This } \\
\text { Cancer }\end{array}$ \\
\end{tabular} & 96 & $29 \%$ & 15 & $16 \%$ & 4 & $11 \%$ & 2 & $50 \%$ \\
\hline & $\begin{array}{c}\text { Unknown/ } \\
\text { Indeterminate }\end{array}$ & 14 & $4 \%$ & 6 & $43 \%$ & 31 & $82 \%$ & & \\
\hline
\end{tabular}

such as The Cancer Genome Atlas (TCGA) and the International Cancer Genome Consortium (ICGC) [9], the field of cancer genomics continues to advance at an extraordinarily rapid pace. Using this specific tool, we first examined and identified DNA copy number gains in chromosome 4p16.3, 1p34.2, 12q15, 1q21.3, 10p15.1, 19q12, 8q22.2, 11q13.3, 3p25.2, 1q23.3, and 6p22.3 ( $Q$ value above 9.00 E10-3) in bladder cancer patients. We further analyzed the chromosome 6p22 locus across cancer of 11 different origins. To our great interest, the chromosomal 6p22 amplification was highly prevalent in bladder cancer patients (18\%) compared to other cancer types (Fig. 1A). Further examination of this region of amplification revealed eight known genes (ID4, MBOAT1, E2F3, CDKAL1, Sox4, LINC00340, PRL, and HDGFL1) (Fig. 1B). Moreover, RNA-seq results showed that CDKAL1, E2F3 and Sox4 are highly expressed in patients with the chromosomal 6p22 amplification (Fig. 1C). To validate this finding in our patients, we performed fluorescent in-situ hybridization (FISH) using RP11 clones from the RPCI bacterial artificial chromosomal (BAC) library in TCC-UB tissue microarrays (TMA) 
(Supplemental Table 1). Seven different FISH probe sets at 1 to $2 \mathrm{Mb}$ apart spanning the region chr6:17,627,692$25,167,325$ were used to define the minimal region of amplification to just over $2 \mathrm{Mb}$ as defined by the probe set RP11-239H6 (chr6:20,212,318-20,294,706) and RP11-607F22 (chr6:22,276,349-22,290,957). A complete list of all BAC clones and probe design are listed in Supplementary Table 1 and Supplementary Fig. 1a. A typical positive chromosomal 6p22 amplification FISH image is presented in Supplemental Fig. 1b.

In our cohort of TCC-UB, the $6 \mathrm{p} 22.3$ amplicon was amplified in $17 \%(62 / 364)$ of all patients (predominantly white), but with a much higher rate of $32 \%(12 / 38)$ in the much smaller subset of African Americans $(p=0.0395)$ (Table 1). A comparison of $6 \mathrm{p} 22.3$ amplification in females $(15 \% ; 13 / 87)$ versus males $(18 \% ; 49 / 277)$ (t-test $\mathrm{p}=0.45)$, age $<60(19 \% ; 15 / 81)$ versus age $>60$ $(17 \% ; 47 / 283)(\mathrm{p}=0.96)$, and smokers $(17 \% ; 446 / 275)$ versus non-smokers $(13 \% ; 8 / 60)(\mathrm{p}=0.68)$ showed no major differences. The results of $6 \mathrm{p} 22.3$ analysis with the standard pathological factors of tumor grade, depth of invasion (pT), and nodal metastatic disease $(\mathrm{pN})$ supported that amplification is associated with a more aggressive phenotype. In regard of tumor invasion, there is a major difference in the rate of 6 p22.3 amplification between superficial TCC-UB $(9 \% ; 13 / 137)$ and muscleinvasive TCC-UB $(22 \% ; 49 / 227)(\mathrm{p}=7.2-04)$. Part of this difference is due to the near lack of $6 \mathrm{p} 22.3$ amplification in low-grade superficial TCC-UB $(2 \% ; 1 / 47)$ versus a much higher rate in high-grade superficial TCC-UB (13\%; 12/93). An association of 6 p22.3 amplification with tumor depth of invasion is also noted in muscle-invasive TCCUB $(p=0.12)$, where stage II showed 14\% (7/51) amplified versus a rate of $24 \%(42 / 176)$ for stage III/IV. This marker of aggressive phenotype is also apparent in the evaluation of nodal metastatic disease as the rate of $6 \mathrm{p} 22.3$ amplification in $\mathrm{pN}>1(32 \% ; 27 / 85)$ was higher than for pN0 patients $(16 \% ; 18 / 112)(\mathrm{p}=0.05)$. Interestingly, the rate of $6 \mathrm{p} 22.3$ amplification in $\mathrm{pN}>1$ patients $(40 \% ; 19 / 48)$ was more than twice that in $\mathrm{pN} 1(21 \% ; 8 / 38)$ patients $(\mathrm{p}=0.05)$.

For the 181 muscle-invasive TCC-UB patients who underwent a cystectomy for curative intent, there was no significant association of amplification $(35 / 181 ; 19.2 \%)$ with survival (log-rank $\mathrm{p}=0.438$ ); However, the aggressive phenotype associated with high $\mathrm{pT}$ and $\mathrm{pN}$ stage resulted in more cases that were never disease-free (chi-square $\mathrm{p}=0.032, \mathrm{OR}=2.65$ ). In summary, these results indicate that 6 p22.3 amplification is an early event in tumor progression. Although not associated with survival, it
A

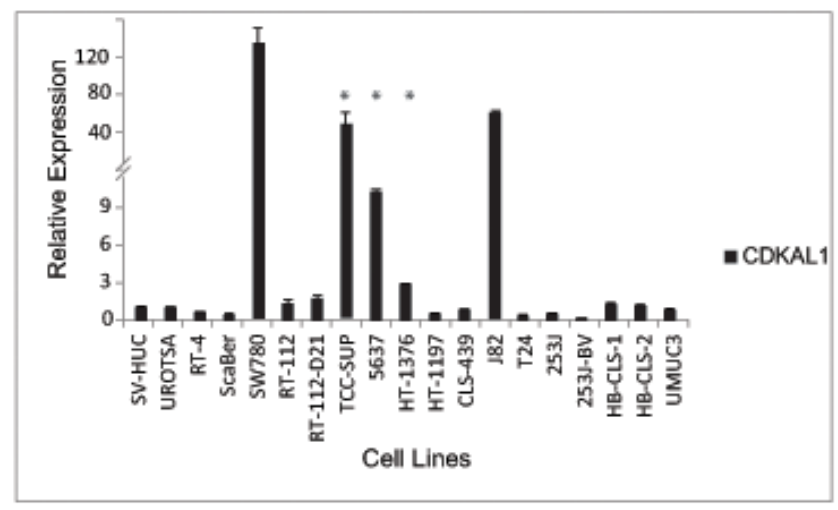

C

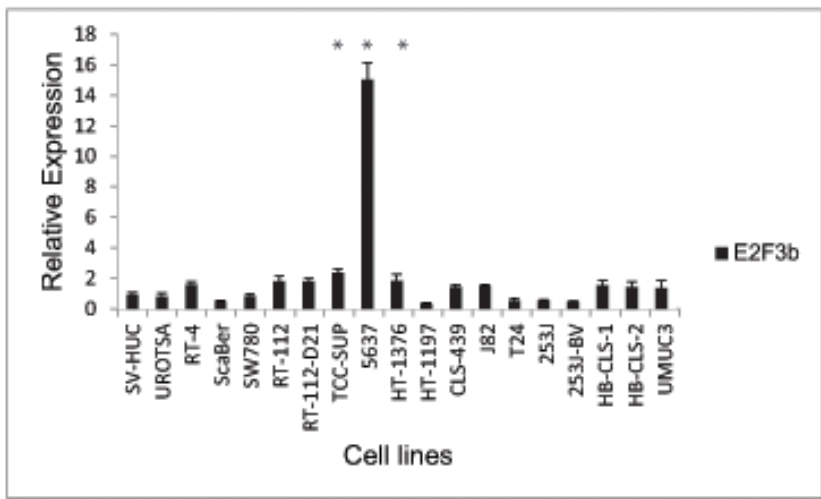

B

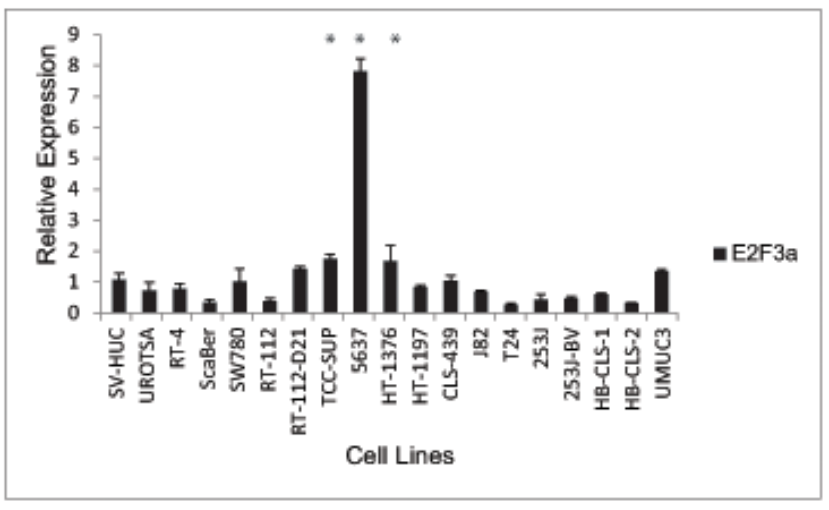

D

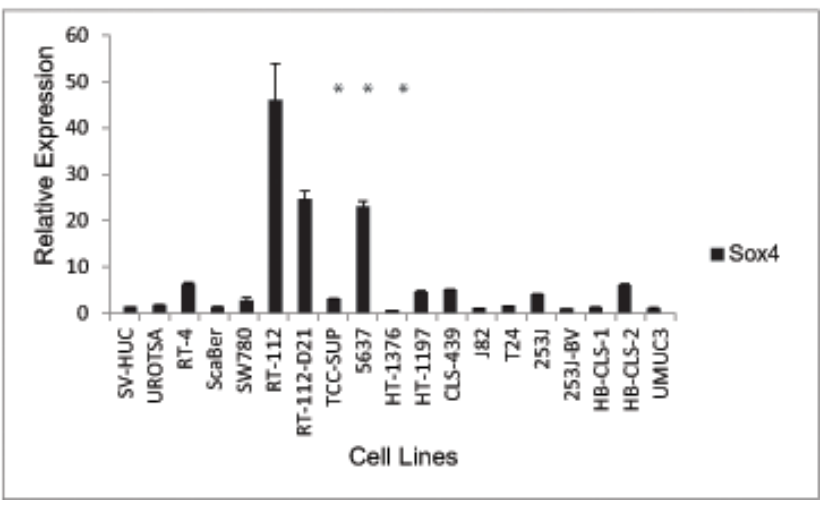

Figure 2: Candidate gene expression in bladder cancer cells. CDKAL1 (A), E2F3a (B), E2F3b (C) and sox4 (D) expression by qRT-PCR in a panel of bladder cancer cell lines. E2F3, sox4 and CDKAL1 are highly expressed in chromosomal-6p22-ampliconcontaining 5637 cells. (*, 6p22-amplicon-containing cell) 
could potentially serve as a biomarker of extensive disease prior to cystectomy.

Among those with high grade superficial bladder cancers, African Americans had a higher percent with 6p22.3 amplification, compared to Whites (18\% compared to $12 \%$ ). African Americans, compared to Whites had a higher percent with the 6 p22.3 amplification among those with both non-muscle invasive and muscle invasive disease. Among those with muscle invasive disease, African Americans, compared to Whites had a higher percent with the 6 p22.3 amplification among those with muscle invasive cancer stage II (17\% vs. 13\%), III (33\% vs. $20 \%)$, and IV (86\% vs. $21 \%)$ and nodal stages pN0 (40\% vs. $15 \%)$, $\mathrm{pN} 1(67 \%$ vs. $17 \%)$, and $\mathrm{pN}>1(67 \%$ vs. $37 \%)$.

\section{Characterization of chromosomal $\quad 6$ p22 amplification in bladder cancer cell lines.}

To further characterize the chromosomal $6 \mathrm{p} 22$ amplification, we performed FISH analysis in a collection of papillary bladder cancer cell lines as well as muscleinvasive bladder cancer cell lines. As a result, we identified three muscle-invasive bladder cancer cell lines (5637, TCC-SUP and HT1376) that contain amplification of the $6 \mathrm{p} 22$ region (Supplemental Table 2). We also performed qRT-PCR to test the gene expression levels in these bladder cancer cell lines. It was found that E2F3a, E2F3b, CDKAL1 and Sox 4 were also highly expressed in the 6p22-amplified 5637 cells (Fig. 2), but the E2F3a and E2F3b mRNA levels in TCC-SUP and HT-1376 cells were similar to those in the control of non-6p22-amplifiedcells. We also noted gene expression values that were not correlated with the presence/absence of the chromosomal 6p22 amplification. For example, high expression of CDKAL1 was found in SW780 and J82 cells and high expression of Sox 4 in RT-112 and RT-112-D21 cells, none of which contain the amplified $6 \mathrm{p} 22$ region.

E2F3 belongs to the E2F transcription factor family. Most E2F proteins associate with a DP protein and form hetero-dimeric complexes that bind to DNA in a sequence specific manner [10]. E2F3 encodes two proteins (E2F3a and E2F3b) through the use of alternative promoters and different 5'-coding exons [11]. During
$\mathrm{G} 1 / \mathrm{S}, \mathrm{Rb}$ protein becomes hyperphosphorylated, leading to the release of E2F3 and its target gene expression [12]. We first examined the E2F3 protein level in a panel of bladder cancer cell lines (Fig.3). Indeed, we detected high level of E2F3 protein in the $6 \mathrm{p} 22$-amplified cells. To further investigate the role of E2F3 in cell proliferation, we designed specific shRNAs against E2F3a, E2F3b and both, and confirmed their knockdown efficiency by immunoblotting (Fig. 4a). Knockdown of E2F3a strongly inhibited cell proliferation, as well as the knockdown of E2F3b and both E2F3 isoforms (Fig. 4a). In contrast, knockdown of CDKAL1 and Sox 4 in 5637 cells had no obvious effect on cell proliferation (Fig. 4b, c). To further confirm that the inhibited cell proliferation induced by E2F3 knockdown is dependent on chromosomal 6p22 amplification, we repeated the experiment in two other cell clines, 253J and T24, that do not contain the chromosomal 6p22 amplification (Supplemental Fig. 3). In contrast, knockdown of E2F3 failed to inhibit cell proliferation in these cell lines, indicating that the above role of E2F3 is dependent on the presence of chromosomal $6 \mathrm{p} 22$ amplification, and perhaps further through an "oncogene addiction" [13-15] mechanism.

\section{Identification of common and distinctive targets of E2F3}

To identify the specific genes regulated by E2F3a and $\mathrm{E} 2 \mathrm{~F} 3 \mathrm{~b}$, we carried out a gene expression profile analysis using Illumina Human HT-12 v4 Expression BeadChips. We used 5637 cells treated with shGFP as a control and compared with those under the condition of E2F3a or E2F3b knockdown to reveal gene expression alterations. A 1.45-fold change and statistical value below 0.05 was used as cut-off in our analyses. Of note, we identified 249 genes that were up-regulated and 322 genes that were down-regulated in response to both E2F3a and E2F3b knockdown; 460 genes that were up-regulated and 473 genes that were down-regulated specifically upon knockdown of E2F3a; as well as 553 genes that were up-regulated and 554 genes that were downregulated specifically upon knockdown of E2F3b (Fig. 5a; Supplemental Table 3).

We then focused on those genes that changed

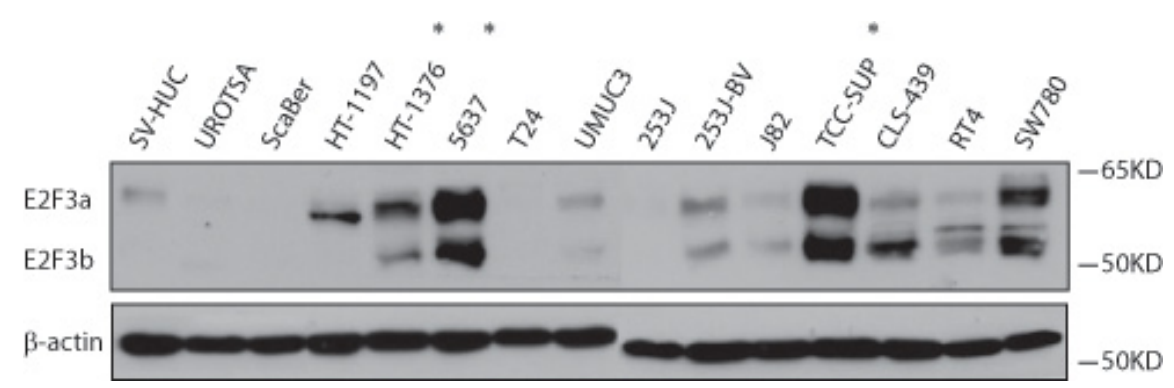

Figure 3: E2F3a and E2F3b protein expression in bladder cancer cells. Increased E2F3a and E2F3b protein level as revealed by immunoblot in 6p22-amplicon-containing 5637 and TCC-SUP cells. (*, 6p22-amplicon-containing cell) 
in response to the knockdown of E2F3a, as shE2F3a elicited a most pronounced phenotype. Among these candidate genes, we identified CCND1 as specifically down-regulated in response to shE2F3a. In addition, we found some signaling pathway changes in the presence of shE2F3a (Fig. 5b). For example, we detected changes of genes involved in the focal adhesion pathways, such as RAC1, ITGA5, ITGB5, COL5A2 and COL8A1; the interleukin and MAPK pathways, such as IL6, IL1A, IL1B, IL7R, MAPK1 and MAPK13; and the tyrosine receptor kinase pathways, such as $\mathrm{HB}-\mathrm{EGF}$, PI3KCD, PIK3CG, IRS1 and IRS2. Our gene expression profiling data demonstrated that E2F3 is not only involved in the classic cell cycle regulation, but also in other signaling pathways and cellular processes that regulate cell migration and invasion.

\section{DISCUSSION}

In the present study, we found that chromosomal
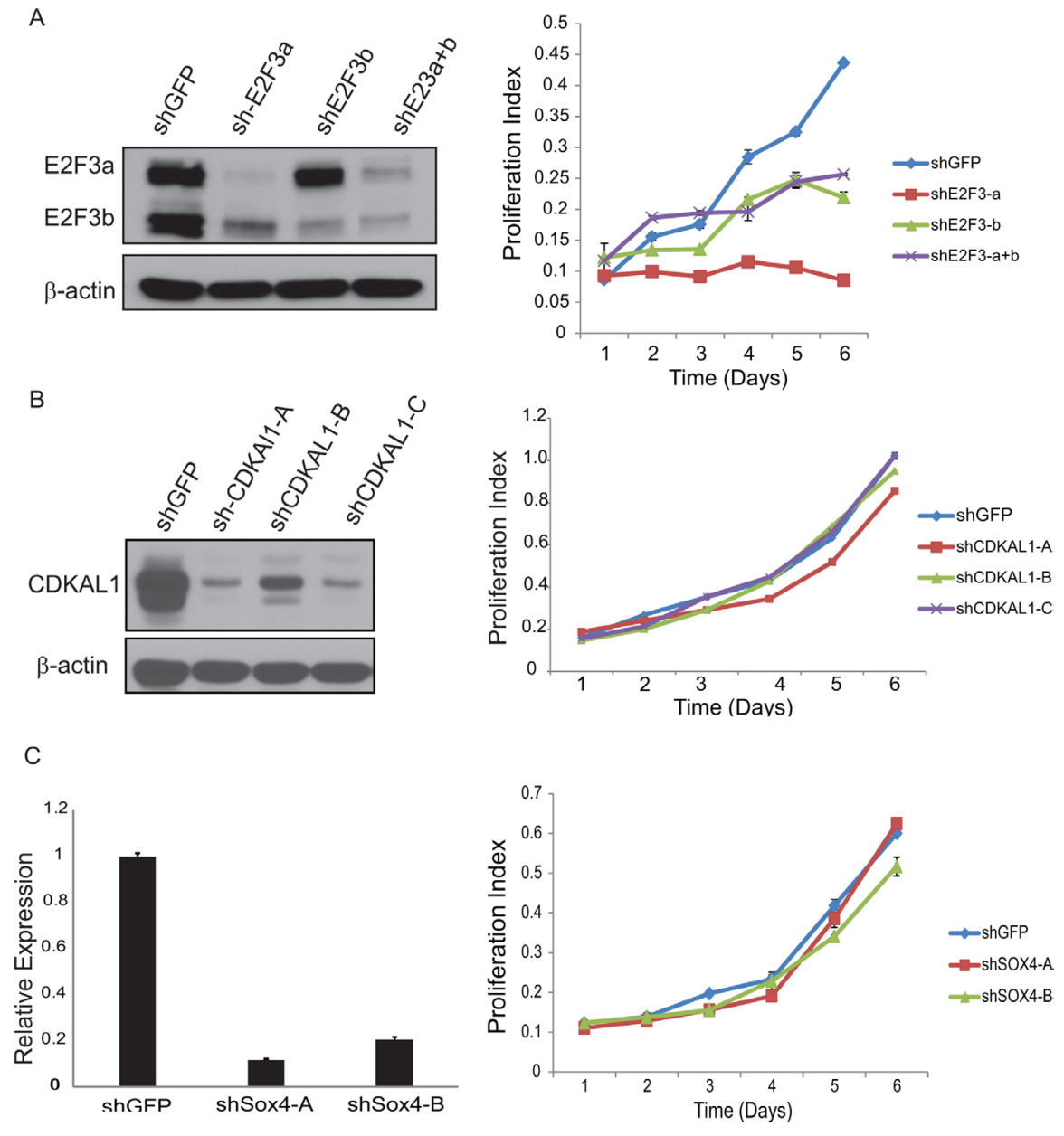

Figure 4: Knockdown of E2F3 inhibits cell proliferation. A) Knockdown of E2F3 inhibits 5637 cell proliferation. Efficient knockdown of E2F3a, E2F3b and E2F3 as revealed by immunoblot ( $\beta$-actin was used as a loading control) and cell proliferation examined by MTT assay. B) Knockdown of CDKAL1 has no effect on 5637 cell proliferation. Efficient knockdown of CDKAL1 as revealed by immnoblot ( $\beta$-actin was used as a loading control) and cell proliferation examined by MTT assay. C) Knockdown of Sox4 has no effect on 5637 cell proliferation. Efficient knockdown of Sox 4 as revealed by qRT-PCR and cell proliferation examined by MTT assay.

6p22.3 amplification is specifically present in bladder cancer as compared to other cancer types in the TCGA dataset. In our cohort of TCC-UB, we found that chromosome 6p22.3 is frequently amplified in the muscle-invasive subset and has significant correlation to tumor stage. As a potential biomarker in TCC-UB, 22.3 amplification would provide risk assessment for vanced $\mathrm{pT}$ or $\mathrm{pN}$ stage in the pre-cystectomy biopsy setting where the only information provided in the typical surgical pathology report is presence or absence of muscle pT or pN stage TCC-UB and the significant morbidity and low rate of mortality associated with cystectomy, there is a clinical need to identify high-stage disease at the time of diagnosis.

Further gene expression profiling analyses revealed candidate oncogenes in a collection of bladder cancer cell lines. For example, proliferation advantage of the 5637 bladder cancer cell line is highly dependent on the $6 \mathrm{p} 22.3$ amplicon, in particular, the E2F3 gene within the locus, and 
knockdown of E2F3a or E2F3b significantly reduced cell proliferation. However, knockdown of Sox 4 and CDKAL1 had minimal effect on cell proliferation [16-19]. Still, we could not rule out other potential functional effects that may result from the amplification of Sox 4 and CDKAL1. For example, elevated Sox 4 expression has been reported in a wide variety of tumors, including leukemia, colorectal cancer, lung cancer and breast cancer, suggesting a fundamental, yet not necessarily pro-proliferation, role in the development of these malignancies [20]. Indeed, in many cancers, deregulated expression of Sox 4 has been associated with obscured tumor cell growth, survival, apoptosis, invasion or metastasis through the epithelialto-mesenchymal transition (EMT) mechanism [20]. In addition, it was recently shown that sox 4 potentiates the RAS-induced tumorigenesis in non-transformed breast cells and reduces the potential of highly metastatic derivatives of breast cancer [21]. Moreover, Tiwari et al recently demonstrated that Sox 4 is a master regulator of epithelial-mesenchymal transition through controlling Ezh2 expression and epigenetic reprogramming [22]. Further studies are warranted to clarify the functional effects of Sox4 in 6p22.3-amplified TCC-UB.

"Oncogene addiction," a term first coined in 2000 by Bernard Weinstein, reveals a possible "Achilles' heel" within the cancer cell that can be exploited therapeutically $[13,14]$. In its simplest embodiment, oncogene addiction refers to the curious observation that a tumor cell, despite its plethora of genetic alterations, can seemingly exhibit dependence on a single oncogenic pathway or protein for its sustained proliferation and/or survival [15]. Our current study revealed that $6 \mathrm{p} 22$ is amplified in more than $20 \%$ of TCC-UB and represents a potential relatively common target in this tumor type. Given the factorial difficulty in targeting transcription factors, identification of the key downstream targets of E2F3 may serve as an

A.
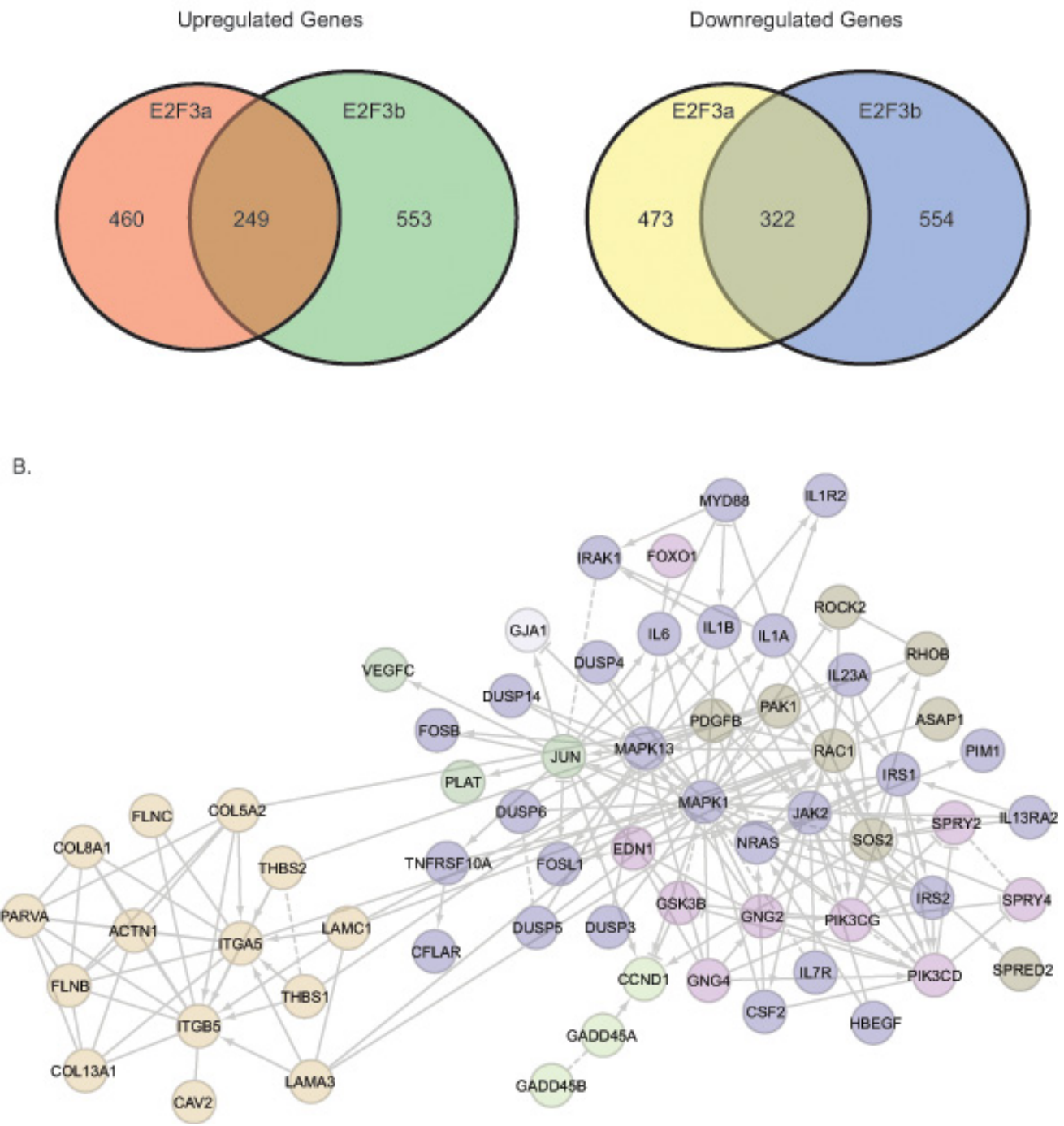

Figure 5: Gene expression profiling reveals common and distinctive E2F3a and E2F3b regulated genes. A) Venn diagram showing common and unique up-regulated and down-regulated genes in response to knockdown of E2F3a or E2F3b. B) Signaling module components identified by integrative bioinformatics and pathway analyses in response to E2F3a knockdown. 
intriguing therapeutic alternative. Of particular note, in our gene expression array analysis, we identified CCND1 as a potential target of E2F3. CCND1 is a key cell cycle regulator specifically responsible for the G1-to-S phase progression [23, 24]. Binding of cyclin D1 to cyclindependent kinase (CDKs) leads to the phosphorylation of retinoblastoma protein $(\mathrm{pRb})$, and subsequently triggers the release of E2F transcription factors to allow the G1to-S phase progression of the cell cycle. It will thus be of interest to test whether inhibition of CCND1 and CDK4/6 may reverse the oncogenic effect elicited by E2F3 amplification in TCC-UB with amplified $6 \mathrm{p} 22$.

We also found racial differences in the $6 \mathrm{p} 22$ amplification. At each stage African Americans had higher percentage with $6 \mathrm{p} 22$ amplification. This is very interesting because African Americans have a lower incidence of and higher mortality from bladder cancer compared to Whites [25-29]. Among this cohort African Americans were diagnosed with more aggressive disease and higher percent with 6p22 amplification. More research is needed to determine whether $6 \mathrm{p} 22$ amplification is associated with the reported racial differences in bladder cancer grade and stage of disease at diagnosis and survival outcomes [25-27, 29]. Furthermore in this cohort (at least in a subset of the cohort) African Americans were more likely to be smokers, more research is required to determine the interaction between smoking, 6p22 amplification and race with regards to bladder cancer aggressiveness and survival.

In summary, we have provided evidence that E2F3 in 6 p22-amplified bladder cancer is a potential oncogene of importance. Future studies are needed to determine the interactions of E2F3 with other genes in the $6 \mathrm{p} 22$ amplicon such as SOX4. In the same manner, other potential oncogenes, such as CCND1, not in the 6 p22 amplicon but yet frequently altered in bladder cancer, could have additional interactions.

\section{METHODS}

\section{Patient Validation Cohort}

Patients included in this study (total 335) were diagnosed and treated with transitional cell carcinoma of the urinary bladder (TCC-UB) between July 1970 and April 2011 at the Roswell Park Cancer Institute. Selection of patients included all in this time period with adequate material in the RPCI archival bank, i.e., adequate material for tissue microarray (TMA) construction and follow-up in the RPCI Tumor Registry. The RPCI Institutional Review Board gave approval for this study. The median patient age at first diagnosis was 69 years (average 68; range 36-91) with 263 males and 72 females. The majority (280 of 335; $81 \%$ ) was either a current smoker at the time of diagnosis, or reported a history of smoking. The majority (325 of $335 ; 97 \%$ ) were white, with the remainder of the cohort consisting of 9 African Americans and 1 American Indian. To more specifically describe this cohort of 335 patients, they were then divided into two general cohorts as either superficial TCC-UB (pTa, Tis, T1) or muscle-invasive TCC-UB (>pT2).

\section{Fluorescent In-situ Hybridization (FISH) for 6p Amplification}

The $6 \mathrm{p}$ amplicon was evaluated by FISH using RP11 clones from the RPCI bacterial artificial chromosomal (BAC) library. A complete list of all BAC clones and probe design are listed in Supplementary Table 1. All FISH was done in a CLIA-certified NYS inspected laboratory using standard operating procedures on a manual platform. Specimens were evaluated with the Olympus BX51 microscope (Olympus Optical Company, LTD., Japan) under oil immersion at x100 magnification using the recommended filters. For each case the probe of interest was evaluated in at least 60 non-overlapping interphase tumor nuclei. Cells with no signals or with signals of only one color were disregarded. Tumors cells displaying at least 6 up to 10 signals of interest in one or more microclusters in the majority of tumors cells $(>50 \%)$ were considered consistent with low-level amplification at that genomic coordinate. Tumors cells displaying $>10$ signals of interest in one or more microclusters in the majority of tumors cells $(>50 \%)$ were considered consistent with high-level amplification at that genomic coordinate. Tumors cells displaying 6 or more intact signals but without microclusters in the majority of tumors cells $(>50 \%)$ were considered consistent with polysomy and not amplification at that genomic coordinate. Cases with isolated large or bilobed cell tumor nuclei and increased signals of interest with or without microclusters were not considered as amplification as we consider these to be cells in mitosis with an extended chromatin pattern.

\section{Tissue Microarrays}

Three 1-millimeter tissue cores from formalin-fixed paraffin embedded donor blocks were precisely arrayed into a new recipient paraffin block, including tumor specimens as well as controls. Specimens for controls within the TMA consisted of multiple cores of normal tissue from 10 different organs, including heart, colon, kidney, adrenal, ovary, myometrium, brain, thyroid, lung, and prostate, representing slightly more than $20 \%$ of all the cores in a TMA. 


\section{Cell Culture}

Human bladder cancer cell lines, 5637, TCC-SUP, HT-1376, 253J and T24 cells were cultured as described [19]. Transfection was performed using X-tremeGENE 9 DNA Transfection Reagent following the manufacturer's protocol (Roche). Packaging of lentivirus, cell transduction and drug selection were performed following standard protocols. For knockdown experiments, control shRNA was designed to target green fluorescent protein (GFP), a gene not expressed endogenously. shRNA hairpins targeting human E2F3a, E2F3b and E2F3 were cloned into pLKO.1 shRNA vector. The target sequences are listed (in the 5'-3' direction): shE2F3a: 5'-GCGTACATCCAGATCCTCACA-3', shE2F3b 5'-GGAAATGCCCTTACAGCAGCA-3', shE2F3 $(\mathrm{a}+\mathrm{b})$ : 5' AAGACCAAACTGTTATAGTTG-3', shSOX4-A: 5'-AGCGACAAGATCCCTTTCATT-3', shSOX4-B: 5'-CCTTTCTACTTGTCGCTAAAT-3', shCDKAL1-D1: 5'-CCTGAACAGTTGCACTGTAAA-3', shCDKAL1-D2: 5'-CGATTGGATTTGCCGAAGATT-3', shCDKAL1-D3: 5'-AGTTGTGGAGGAGACAATTAA-3'.

\section{Real-time RT-PCR}

For RNA preparation and qRT-PCR, RNA was extracted using the Trizol reagent (Invitrogen). cDNA synthesis was performed using the First-Strand cDNA Synthesis Kit (GE Healthcare) and quantitative realtime RT-PCR was performed using Power SYBR Green PCR Master Mix (Invitrogen). Sequences of the qPCR primer pairs (in the 5'-3' direction) are as follows: E2F3a-F: 5'-GCGCGTACATCCAGATCC-3', E2F3a-R: 5'-TTGGAGGGAGGAGGAACAG-3'; E2F3b-F: 5'-GCCCTTACAGCAGCAGGC-3', E2F3b-R: 5'-GGACTATCTGGACTTCG-3'; CDKAL1-F: 5'-TGGATTTGCCGAAGATTAG- 3', CDKAL1-R: 5'-TTTCCTCTGGCGTGTTTAG- 3 '; $\quad$ SOX4-F: 5'-ACCGGGACCTGGATTTTAAC-3', SOX4-R: 5'-AAACCAGGTTGGAGATGCTG-3'; $\beta$-actin-F: 5'-CCAACCGCGAGAAGATGA-3', $\quad \beta$-actin-R: 5'-CCAGAGGCGTACAGGGATAG-3'. Measurements were performed in triplicate and standardized to the levels of $\beta$-actin.

\section{Cell Proliferation (MTT) Assay}

Five thousand to eight thousand bladder cancer cells were plated onto 96 wells plate. Plates were harvested daily. 20 ul of $5 \mathrm{mg} / \mathrm{ml}$ MTT (3-(4,5-Dimethylthiazol-2yl)-2,5-diphenyltetrazolium bromide, a tetrazole) were added to each well, incubated for 3.5 hours in $37{ }^{\circ} \mathrm{C}$, and carefully removed without disturbing the cells. $150 \mathrm{ul}$
MTT solvent (4 mM HCl, 0.1\% Nondet P-40 (NP40), all in isopropanol was added into each well and plate shaken at room temperature for $15 \mathrm{~min}$. Absorbance was read at $590 \mathrm{~nm}$ with a reference filter of $620 \mathrm{~nm}$.

\section{Amplification, labeling and BeadChip hybridization of RNA samples:}

The Illumina TotalPrep RNA Amplification Kit (Ambion) was used to transcribe 200ng of total RNA according to the manufacture's recommendation(s). Approximately, 700ng of cRNA was hybridized at $58^{\circ} \mathrm{C}$ for 16 hours to Illumina HumanHT-12 v4 Expression BeadChips (Illumina) and scanned using an Illumina BeadArray Reader and Bead Scan Software.

\section{Microarray data processing and pathway analysis}

Data processing was performed with GenomeStudio version 2011.1 and the R Language and Environment for Statistical Computing (R) 2.13.2 in combination with Bioconductor 2.10 [30]. The Bioconductor lumi package [31] was used for quality control. The normalizations executed by Illumina GenomeStudio were all applied to the expression values on the original scale. If background adjustment was performed, we used the standard background correction offered by BeadStudio (bg *); however, data was subsequently normalized using variance stabilization (VSN) and quantile normalization across samples using the functions in the 'lumi' Bioconductor (available at www.bioconductor.org). Differentially expressed genes were selected using a modified t-statistic with $15 \%$ of the standard deviation percentile as the fudge constant and fold change ratios, followed by BenjaminiHochberg testing procedure for controlling FDR (False Discovery Rate) of $5 \%$ and permutation FDR of $7 \%$, respectively. All methods used are implemented in the $R$ packages lumi [31].

To investigate functional network and gene ontology relationships, candidate genes were first categorized using the Panther classification system [32]. For each molecular function, biological process or pathway term in PANTHER, the genes associated with that term were evaluated according to the likelihood that their numerical values were drawn randomly from the overall distribution of values. The Mann-Whitney U Test (Wilcoxon RankSum Test) was used to determine the P-value relative to overall list of values that were input (nominal $\mathrm{P}$ value= $0.05)$.

Ingenuity Pathway Analysis (IPA) software was used to perform pathway analysis and describe functional relationships between gene products based on known interactions in the literature (http://www.ingenuity.com). Focus genes were overlaid onto a global molecular network developed from direct and/or indirect interactions, 
and sub-networks were algorithmically generated based on their connectivity. Pathways with a score greater than 4 $(p<0.0001)$ were combined to form a composite network representing the underlying biological process.

\section{Statistical Analysis}

Association between clinical/histological covariates and $6 \mathrm{p} 22$ amplification was tested using two-sample t-tests for the equality of proportions. Survival time associations were tested with a log-rank test. Statistical analysis of data was performed using the SPSS statistics software package (SPSS, IL). All results are expressed as mean \pm SD.

\section{ACKNOWLEDGEMENTS}

This work was supported by Roswell Park Cancer Institute and National Cancer Institute (NCI) grant \#P30 CA016056 (to J.Z) and an award from the Roswell Park Alliance Foundation (to J.Z); The National Cancer Institute (NCI) grant CA16056-36; CA067267-18 (to J.C).

\section{REFERENCES}

1. Bakkar AA, Wallerand H, Radvanyi F, Lahaye JB, Pissard S, Lecerf L, Kouyoumdjian JC, Abbou CC, Pairon JC, Jaurand MC, Thiery JP, Chopin DK, de Medina SG. FGFR3 and TP53 gene mutations define two distinct pathways in urothelial cell carcinoma of the bladder. Cancer Res 2003;63:8108-12.

2. Jebar AH, Hurst CD, Tomlinson DC, Johnston C, Taylor CF, Knowles MA. FGFR3 and Ras gene mutations are mutually exclusive genetic events in urothelial cell carcinoma. Oncogene 2005;24:5218-25.

3. Castillo-Martin M, Domingo-Domenech J, Karni-Schmidt $\mathrm{O}$, Matos T, Cordon-Cardo C. Molecular pathways of urothelial development and bladder tumorigenesis. Urol Oncol 2010;28:401-8.

4. Cordon-Cardo C, Wartinger D, Petrylak D, Dalbagni G, Fair WR, Fuks Z, Reuter VE. Altered expression of the retinoblastoma gene product: prognostic indicator in bladder cancer. J Natl Cancer Inst 1992;84:1251-6.

5. Goebell PJ, Knowles MA. Bladder cancer or bladder cancers? Genetically distinct malignant conditions of the urothelium. Urol Oncol 2010;28:409-28.

6. McConkey DJ, Lee S, Choi W, Tran M, Majewski T, Siefker-Radtke A, Dinney C, Czerniak B. Molecular genetics of bladder cancer: Emerging mechanisms of tumor initiation and progression. Urol Oncol 2010;28:429-40.

7. Bruch J, Schulz WA, Haussler J, Melzner I, Bruderlein S, Moller P, Kemmerling R, Vogel W, Hameister H. Delineation of the $6 \mathrm{p} 22$ amplification unit in urinary bladder carcinoma cell lines. Cancer Res 2000;60:4526-30.

8. Santos GC, Zielenska M, Prasad M, Squire JA.
Chromosome 6p amplification and cancer progression. J Clin Pathol 2007;60:1-7.

9. Cerami E, Gao J, Dogrusoz U, Gross BE, Sumer SO, Aksoy BA, Jacobsen A, Byrne CJ, Heuer ML, Larsson E, Antipin Y, Reva B, Goldberg AP, Sander C, Schultz N. The cBio cancer genomics portal: an open platform for exploring multidimensional cancer genomics data. Cancer Discov 2012;2:401-4.

10. van den Heuvel S, Dyson NJ. Conserved functions of the pRB and E2F families. Nat Rev Mol Cell Biol 2008;9:71324.

11. Leone G, Nuckolls F, Ishida S, Adams M, Sears R, Jakoi L, Miron A, Nevins JR. Identification of a novel E2F3 product suggests a mechanism for determining specificity of repression by Rb proteins. Mol Cell Biol 2000;20:362632.

12. Trimarchi JM, Lees JA. Sibling rivalry in the E2F family. Nat Rev Mol Cell Biol 2002;3:11-20.

13. Weinstein IB. Cancer. Addiction to oncogenes--the Achilles heal of cancer. Science 2002;297:63-4.

14. Weinstein D, Staffelbach D, Biaggio M. Attention-deficit hyperactivity disorder and posttraumatic stress disorder: differential diagnosis in childhood sexual abuse. Clin Psychol Rev 2000;20:359-78.

15. Sharma SV, Settleman J. Oncogene addiction: setting the stage for molecularly targeted cancer therapy. Genes Dev 2007;21:3214-31.

16. Olsson AY, Feber A, Edwards S, Te Poele R, Giddings I, Merson S, Cooper CS. Role of E2F3 expression in modulating cellular proliferation rate in human bladder and prostate cancer cells. Oncogene 2007;26:1028-37.

17. Oeggerli M, Tomovska S, Schraml P, Calvano-Forte D, Schafroth S, Simon R, Gasser T, Mihatsch MJ, Sauter G. E2F3 amplification and overexpression is associated with invasive tumor growth and rapid tumor cell proliferation in urinary bladder cancer. Oncogene 2004;23:5616-23.

18. Oeggerli M, Schraml P, Ruiz C, Bloch M, Novotny H, Mirlacher M, Sauter G, Simon R. E2F3 is the main target gene of the $6 \mathrm{p} 22$ amplicon with high specificity for human bladder cancer. Oncogene 2006;25:6538-43.

19. Hurst CD, Tomlinson DC, Williams SV, Platt FM, Knowles MA. Inactivation of the $\mathrm{Rb}$ pathway and overexpression of both isoforms of E2F3 are obligate events in bladder tumours with 6p22 amplification. Oncogene 2008;27:271627.

20. Vervoort SJ, van Boxtel R, Coffer PJ. The role of SRYrelated HMG box transcription factor 4 (SOX4) in tumorigenesis and metastasis: friend or foe? Oncogene 2012.

21. Zhang J, Liang Q, Lei Y, Yao M, Li L, Gao X, Feng J, Zhang Y, Gao H, Liu DX, Lu J, Huang B. SOX4 induces epithelial-mesenchymal transition and contributes to breast cancer progression. Cancer Res 2012;72:4597-608.

22. Tiwari N, Tiwari VK, Waldmeier L, Balwierz PJ, Arnold 
P, Pachkov M, Meyer-Schaller N, Schubeler D, van Nimwegen E, Christofori G. Sox4 is a master regulator of epithelial-mesenchymal transition by controlling Ezh2 expression and epigenetic reprogramming. Cancer Cell 2013;23:768-83.

23. Nishi K, Inoue H, Schnier JB, Rice RH. Cyclin D1 downregulation is important for permanent cell cycle exit and initiation of differentiation induced by anchoragedeprivation in human keratinocytes. J Cell Biochem 2009;106:63-72.

24. Muller H, Lukas J, Schneider A, Warthoe P, Bartek J, Eilers M, Strauss M. Cyclin D1 expression is regulated by the retinoblastoma protein. Proc Natl Acad Sci U S A 1994;91:2945-9.

25. Lee CT, Dunn RL, Williams C, Underwood W, 3rd. Racial disparity in bladder cancer: trends in tumor presentation at diagnosis. J Urol 2006;176:927-33; discussion 33-4.

26. Underwood W, 3rd, Dunn RL, Williams C, Lee CT. Gender and geographic influence on the racial disparity in bladder cancer mortality in the US. J Am Coll Surg 2006;202:28490.

27. Jacobs BL, Montgomery JS, Zhang Y, Skolarus TA, Weizer AZ, Hollenbeck BK. Disparities in bladder cancer. Urol Oncol 2012;30:81-8.

28. Yee DS, Ishill NM, Lowrance WT, Herr HW, Elkin EB. Ethnic differences in bladder cancer survival. Urology 2011;78:544-9.

29. Madeb R, Messing EM. Gender, racial and age differences in bladder cancer incidence and mortality. Urol Oncol 2004;22:86-92.

30. Gentleman RC, Carey VJ, Bates DM, Bolstad B, Dettling M, Dudoit S, Ellis B, Gautier L, Ge Y, Gentry J, Hornik K, Hothorn T, Huber W, Iacus S, Irizarry R, Leisch F, Li C, Maechler M, Rossini AJ, Sawitzki G, Smith C, et al. Bioconductor: open software development for computational biology and bioinformatics. Genome Biol 2004;5:R80.

31. Du P, Kibbe WA, Lin SM. lumi: a pipeline for processing Illumina microarray. Bioinformatics 2008;24:1547-8.

32. Mi H, Guo N, Kejariwal A, Thomas PD. PANTHER version 6: protein sequence and function evolution data with expanded representation of biological pathways. Nucleic Acids Res 2007;35:D247-52. 